\title{
Controlling anomalous stresses in soft field-responsive systems
}

\author{
D. Reguera, J. M. Rubí, and A. Pérez-Madrid \\ Departament de Física Fonamental-CER on Physics of Complex Systems, Facultat de Física, Universitat de Barcelona, \\ Diagonal 647, 08028 Barcelona, Spain
}

(Received 1 March 2000)

\begin{abstract}
We report a phenomenon occurring in field-responsive suspensions: shear-induced anomalous stresses. Competition between a rotating field and a shear flow originates a multiplicity of anomalous stress behaviors in suspensions of bound dimers constituted by induced dipoles. The great variety of stress regimes includes nonmonotonic behaviors, multiresonances, negative viscosity effect, and blockades. The reversibility of the transitions between the different regimes and the self-similarity of the stresses make this phenomenon controllable and therefore applicable to modify macroscopic properties of soft condensed matter phases.

PACS number(s): 47.50. $+\mathrm{d}, 83.80 . \mathrm{Gv}, 75.50 . \mathrm{Mm}$
\end{abstract}

\section{INTRODUCTION}

Field-responsive systems constitute a class of soft condensed matter systems undergoing significant responses leading to important macroscopic changes upon application of an external field [1-3]. This characteristic has been used in many applications and may become useful in the implementation of different devices [4]. Electrorheological and magnetorheological fluids, ferrofluids and magnetic holes are typical examples of field-responsive systems that have been subject of many recent investigations [5-9].

These systems consist essentially in two phases, one is a dispersion of smart active units, whereas the other is a liquid, or more generally a soft phase, practically inactive to the action of the field. The mechanical response of such units to the applied field depends on their nature. If the particles bear permanent dipoles, they induce stresses in the liquid phase during their reorientation process even in the single-particle domain. When the dipoles are induced, their dipolar moments are always collinear with the field. Therefore in this case the only way to induce mechanical responses is through the formation of assemblies of particles, which occurs at higher concentrations, when dipolar interactions start to play a significant role. The elementary assembled unit exhibiting mechanical response is a bound pair of induced dipoles (dimer).

Our purpose in this paper is to show that stresses induced by these field-responsive elementary units, the bounded dimers, exhibit a multiplicity of regimes emerging from the nonlinear nature of the dynamics, not observed in other fieldresponsive phases analyzed up to now. The stresses are anomalous as they do not necessarily vary monotonously with the characteristic parameters, and reversible as their appearance is not subjected to intrinsic structural changes of the system. This peculiar property has an important consequence: it can be used to control the induction of stresses in the solvent phase.

We have organized the paper in the following way. In Sec. II, we introduce the model describing the dynamics of the system. Section III is devoted to analyzing the stresses generated by the particles, whereas in Sec. IV we discuss the rheology of the suspension. Finally, the last section is intended as a summary of our main results.

\section{THE MODEL}

To illustrate this phenomenon, we consider a twodimensional (2D) model in which the dynamics of the orientation $\varphi$ of the bounded dimer captures the two basic ingredients present in experimental situations, namely a pure rotation caused by the applied field and a term breaking the symmetry of the dynamics that originates from the presence of a shear flow:

$$
\dot{\varphi}=-A(t) \sin [2\{\varphi-\alpha(t)\}]-b \sin ^{2} \varphi,
$$

where the overdot denotes total time derivative. Here we have considered the general case in which the pure rotation is modulated by the frequency $w_{h}$ by

$$
A(t)=w_{c}\left[\cos ^{2}\left(w_{h} t\right)+r^{2} \sin ^{2}\left(w_{h} t\right)\right],
$$

where $w_{c}$ is a characteristic frequency and $r$ denotes its degree of polarization ranging from $r=1$ corresponding to circular polarization to $r=0$ holding for linear polarization; $\alpha(t)$ is a time-dependent phase and $b$ is the shear rate.

Physical realizations of this model are in general the 2D dynamics of a bounded pair of spherical induced dipoles in the presence of a shear flow with velocity profile $b y \hat{\mathbf{x}}$, and of an external rotating field with frequency $\omega_{h}$ and components $H_{x} \cos \left(\omega_{h} t\right) \hat{\mathbf{x}}$ and $H_{y} \sin \left(\omega_{h} t\right) \hat{\mathbf{y}}$. The equation of motion of the rotating dimer Eq. (1) then emerges from balancing out the hydrodynamic torque

$$
T_{h y}=-6 \pi \eta_{0}\left(b \sin ^{2} \varphi+\dot{\varphi}\right)
$$

and the external field torque

$$
T_{M}=-6 \pi \eta_{0} A(t) \sin [2\{\alpha(t)-\varphi\}]
$$

arising from the energy of dipolar interaction

$$
U(\varphi)=\frac{M_{V}^{2}\left[1-3 \cos ^{2}\{\alpha(t)-\varphi\}\right]}{d^{3}} .
$$

In the previous equations, $\eta_{0}$ is the viscosity of the liquid phase, $\alpha(t)=\arctan \left[r \tan \left(w_{h} t\right)\right]$ is the direction of the field, and $M_{V}=V \chi_{e f f} H$ is the induced moment with $\chi_{e f f}$ the 
effective susceptibility and $V$ is the volume of the sphere with diameter $d$. Within this context, the value of the characteristic frequency can be identified with $w_{c}$ $=\chi_{e f f}^{2} H_{x}^{2} V^{2} / 2 \pi \eta_{0} d^{3}$, and $r=H_{y} / H_{x}$. Our model is motivated and extends the work of Skjeltorp et al. [10,11], which studies a magnetic rotor in the absence of the symmetrybreaking term, in the context of the nonlinear dynamics of a bounded pair of magnetic holes. For the particular case $w_{c}$ $=m H_{x} / 6 \pi \eta_{0}$ and $b=0$, Eq. (1) also describes the 2D dynamics of a ferrofluid particle with magnetic moment $m$ in a static magnetic field and a vorticity field $-w_{h} \hat{\mathbf{z}}$, in the absence of noise [12].

The motion of the pair induces stresses in the whole system emerging from the conversion of field torque into tensions in the fluid. In this sense, this process can be viewed as a mechanism of transduction of field energy into stresses whose efficiency is determined by the dynamics. The induced stress is simply the averaged density of hydrodynamic torque:

$$
S=6 \eta_{0} c\left\langle\dot{\varphi}+b \sin ^{2} \varphi\right\rangle
$$

where $c$ represents the volume fraction of dimers. Notice that, as we have employed Stokes law in Eqs. (3) and (6), the applicability of the general model described by Eq. (1) is conditioned to the requirement of small angular Reynolds numbers $R=\dot{\varphi} d^{2} / \nu$, where $\nu$ is the kinematic viscosity [13]. Since the dimers are usually small, the requirement of small angular Reynolds numbers does not constitute a significant restriction of the validity of the model.

\section{DYNAMICS AND STRESSES}

\section{A. Circular polarization}

In order to elucidate the main features of this model, we have solved numerically Eq. (1). In Fig. 1(a) we have depicted the stress as a function of the frequency of the field, for different values of shear rate corresponding to the case of circular polarization $(r=1)$. Since the stress is an homogeneous function $S\left(\lambda w_{c}, \lambda b, \lambda w_{h}\right)=\lambda S\left(w_{c}, b, w_{h}\right)$, its behavior can be analyzed in terms of the scaled quantities $b^{\prime}$ $=b / w_{c}, w_{h}^{\prime}=w_{h} / w_{c}$, and $S^{\prime}\left(w_{h}^{\prime}, b^{\prime}\right)=S / w_{c}$.

In the absence of shear flow $\left(b^{\prime}=0\right)$, the interplay between hydrodynamic and field effects originates two basic dynamic regimes determined by the value of $\left|w_{h}^{\prime}\right|$. When this frequency is smaller than the threshold $\left|w_{h}^{\prime}\right|=1$, the dimer follows the field with a fixed phase-lag and the same angular velocity, performing uniform oscillations. At frequencies higher than the threshold value the system is no longer able to follow the field and undergoes periodic rotations with stops and backward motions (jerky oscillations) [10]. These two modes of motion are manifested in two different regimes of the stress in Fig. 1(a). A linear regime, for $\left|w_{h}^{\prime}\right|<1$, in which the scaled stress is just the frequency of the field and a monotonous decay regime for $\left|w_{h}^{\prime}\right|>1$, where the modulus of the stress decreases due to jerky oscillations. During backward rotations field energy is wasted inducing "wrong sign tensions." When they become as important as forward rotations, which occurs at high frequencies, the net transduction

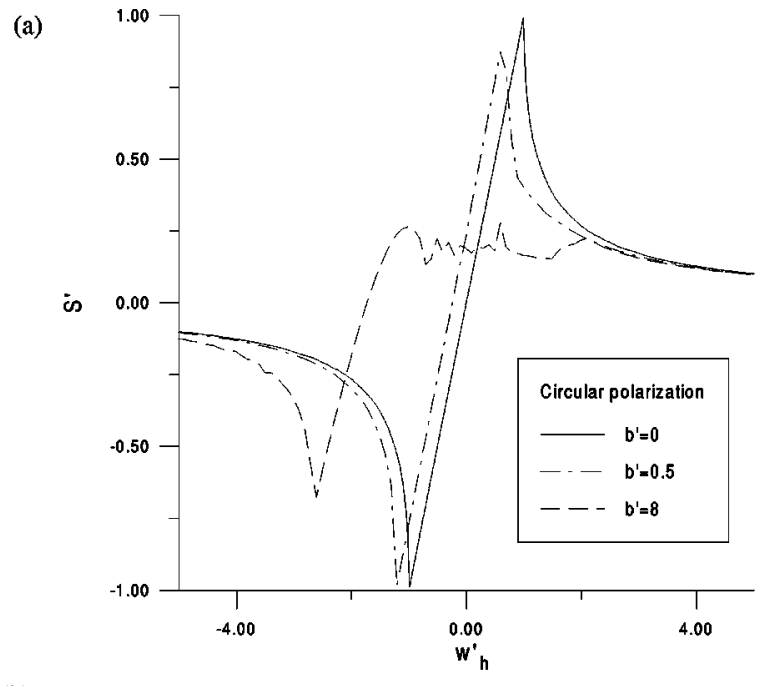

(b)

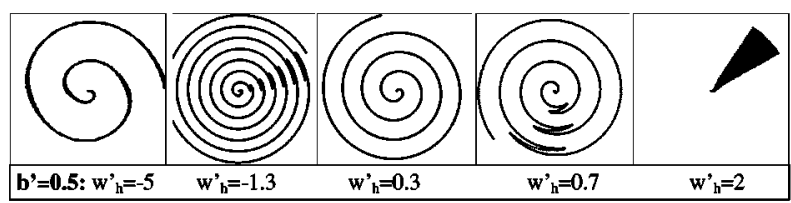

(c)

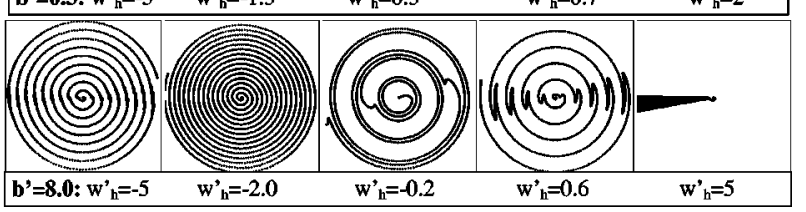

FIG. 1. (a) Scaled stress as a function of the frequency of the field for some representative values of $b^{\prime}$ for the case of circular polarization. Below we include polar plots of $\varphi$ vs time (radially) representing the dynamical regimes for different values of $w_{h}^{\prime}$ and for values of the scaled shear rate (b) $b^{\prime}=0.5$, (c) $b^{\prime}=8.0$.

of energy, and consequently the induced stress, is practically nonexistent.

The presence of a shear flow completely modifies the dynamical response leading to the appearance of a richer phenomenology. The role played by the flow is manifold. On one hand, it breaks the symmetry of the dynamics by fixing a direction of rotation that implies that the property $S^{\prime}\left(w_{h}^{\prime}\right)$ $=-S^{\prime}\left(-w_{h}^{\prime}\right)$, which holds in the absence of shear, is no longer valid. On the other hand, the regimes in which one of the two competing rotational mechanisms, related to the field and to the flow, dominates are intrinsically different. Finally, the presence of the new time scale $b$ is responsible for the appearance of new synchronization mechanisms.

For $b^{\prime}<1$, the strength of the field dominates and the behavior of the stress is similar to that of the case $b^{\prime}=0$ but shifted in frequency by an amount $b^{\prime} / 2$ as one can notice in Fig. 1(a). In Fig. 1(b), we have represented some snapshots of the dynamical modes of rotation corresponding to the different regimes in the stress. Upon increasing $w_{h}^{\prime}$, we can generate the sequence of modes: jerky (first two snapshots)uniform-jerky-localized oscillations. We have found a dynamic transition, from jerky to localized oscillations, at a characteristic positive frequency, which depends on $b^{\prime}$, with its subsequent macroscopic consequences in the stress. Moreover, competition between flow and field involves the breaking of the symmetry of the stress and leads to the decrease of the stress peak at positive frequencies. 


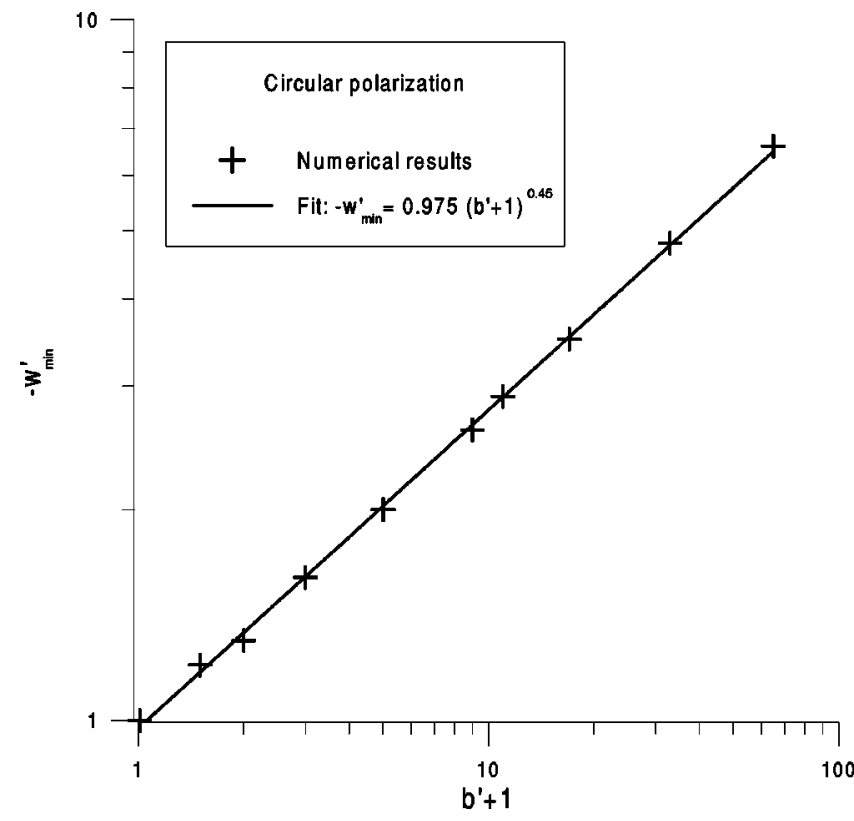

FIG. 2. Scaling law for the frequency corresponding to the negative stress minimum for different values of the shear rate, in the case of circular polarization.

In the opposite case, when $b^{\prime}>1$, the effects of the flow dominate and in this situation, even at frequencies near to zero, the rotation imposed by the field is very different from the one dictated by the shear. As we can see in Fig. 1(a), the positive peak has definitively disappeared and the behavior of the stress is characterized by the development of small multiresonances followed by linear increases and decreases of the stress with slopes $-1,-2 \ldots$ The origin of this behavior is the synchronization of the field and the shear, exciting mode locks of the pair with frequencies ratio $S^{\prime}: w_{h}^{\prime}$ of $1: 1$ or $2: 1$, etc. It is important to highlight that, for every value of the shear rate, stress curves overlap at high and moderate frequencies.

As an illustrative example, in Fig. 1(c) we have depicted some snapshots of the dynamics for $b^{\prime}=8$ corresponding to the different regimes of the stress obtaining a sequence of modes: jerky-uniform-jerky (third and fourth snapshots) localized oscillations, upon increasing $w_{h}^{\prime}$.

It is worth pointing out that the frequency of the negative stress minimum, where transition between linear and jerky oscillations regime occurs, follows a power law in terms of the shear rate: $w_{\min }^{\prime} \sim-\left(b^{\prime}+1\right)^{0.45}$, as it is shown in Fig. 2 .

\section{B. Elliptical polarization}

Even more interesting is the case of elliptical polarization. In Fig. 3(a), we have depicted the stress against frequency for $r=0.5$ and different values of the shear rate. In the absence of shear, the dynamics basically exhibits three different modes as we increase $\left|w_{h}^{\prime}\right|$ : (i) a phase-locked mode, where the system performs modulated [by the term $A(t)$ ] uniform rotations with average frequency $w_{h}^{\prime}$; (ii) a modulated "jerky" oscillations mode above a critical frequency; (iii) and localized oscillations, with null average velocity above another characteristic frequency. These modes are responsible for three different behaviors in the stress observed

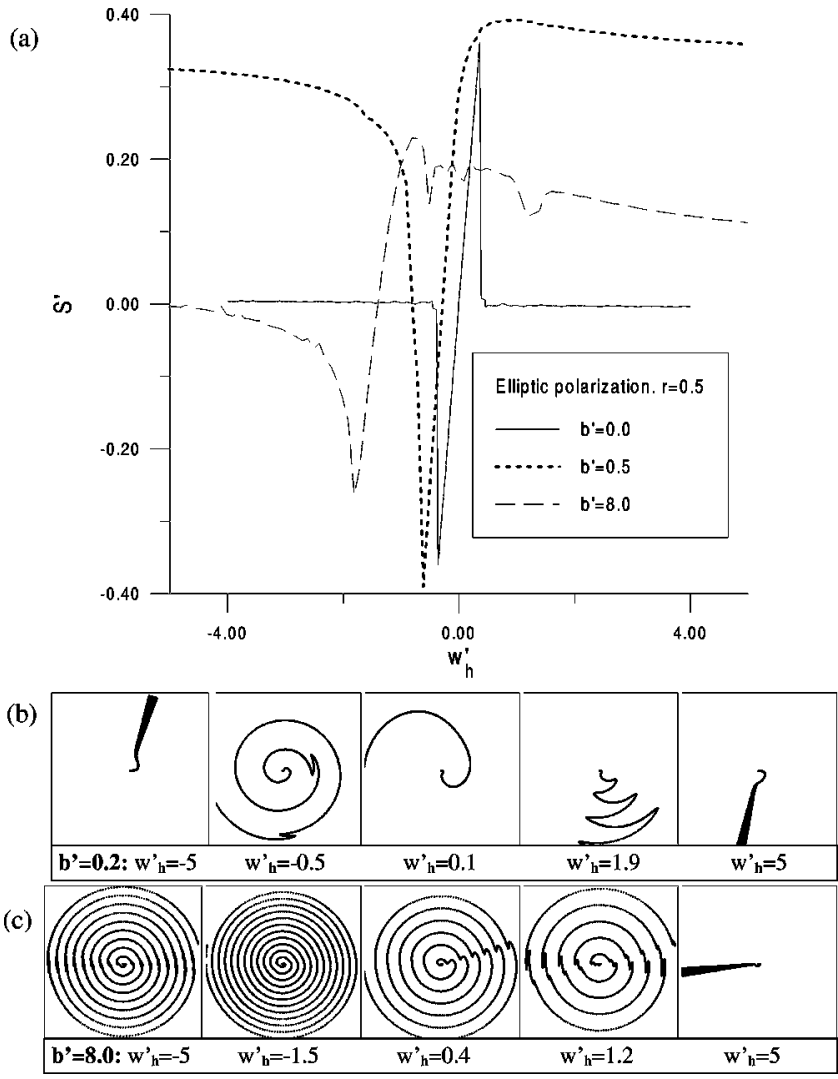

FIG. 3. (a) Scaled stress as a function of the frequency of the field for some representative values of $b^{\prime}$ for the case of $r=0.5$. Below we include polar plots of $\varphi$ vs time (radially) representing the dynamical regimes for different values of $w_{h}^{\prime}$ and for values of the scaled shear rate (b) $b^{\prime}=0.2$ and (c) $b^{\prime}=8.0$.

in Fig. 2(a): a linear regime near $\left|w_{h}^{\prime}\right|=0$, a decay in the modulus due to jerky oscillations, and a nonstress zone when the net rotation vanishes, respectively.

The introduction of the flow changes these regimes significantly. For very low values of $b^{\prime}$, the modes of rotation are slightly modified, as shown in Fig. 3(b); the curve is simply shifted by $b^{\prime} / 2$ in frequencies; and the scaled stress when localization appears is no longer zero but saturates at approximately $b^{\prime}$, positive even for $w_{h}^{\prime}<0$.

At a critical value of the shear rate, the positive stress maximum disappears as shown in Fig. 3(a) for $b^{\prime}=0.5$. Above this value of the shear rate, multiresonances develop near $w_{h}^{\prime} \sim 0$. The critical frequency denoting the transition from uniform to jerky oscillations, corresponding to the position of the minimum of the stress, is shifted following a power law with an exponent near 0.5. Additionally, jerky oscillation mode for negative frequencies persists in a wider range, which causes in turn persistence of the negative stress region at moderate/high frequencies. The dynamic transition from jerky to localized oscillations at negative frequencies is the signature of a change in the sign of the stress.

\section{RHEOLOGY}

When represented as a function of the shear rate, the stress exhibits a wide variety of different anomalous behaviors. This feature contrasts with the monotonous behavior observed in systems inert to the applied field [2]. The exis- 


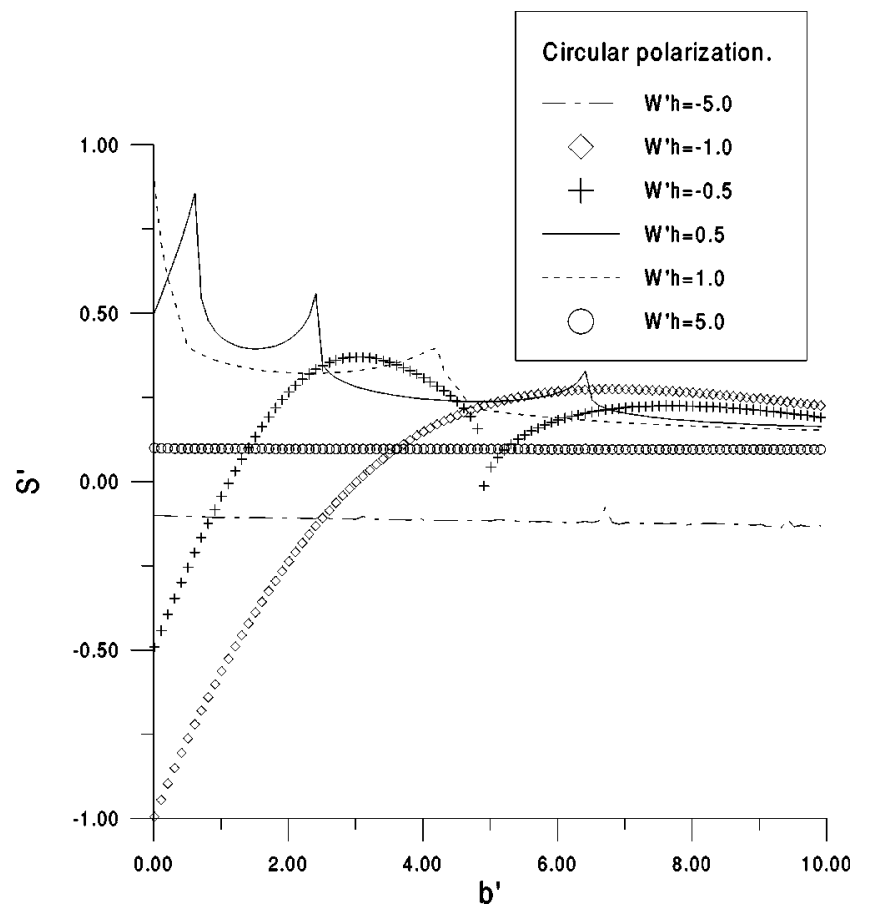

FIG. 4. Scaled stress $S^{\prime}$ as a function of the shear rate $b^{\prime}$ for some values of the frequency of the field $w_{h}^{\prime}$ as well positive (against the rotation induced by the flow) as negative (in the same direction as the rotation of the flow) for the case of circular polarization.

tence of such a rich phenomenology is manifested in Figs. 4 and 5. Their most salient feature is that upon fixing a proper value of the frequency of the field, we can monitor and promote drastic changes in the mechanical response of the system.

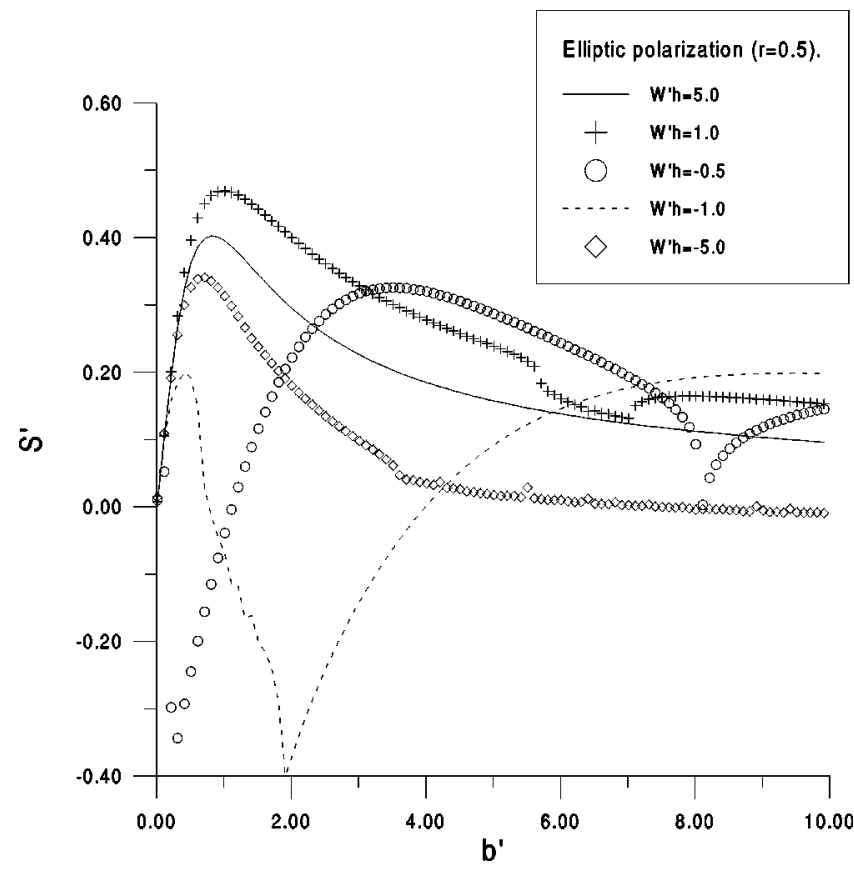

FIG. 5. Scaled stress $S^{\prime}$ as a function of the shear rate $b^{\prime}$ for some values of the frequency of the field $w_{h}^{\prime}$ as well positive (against the rotation induced by the flow) as negative (in the same direction as the rotation of the flow) corresponding to $r=0.5$.

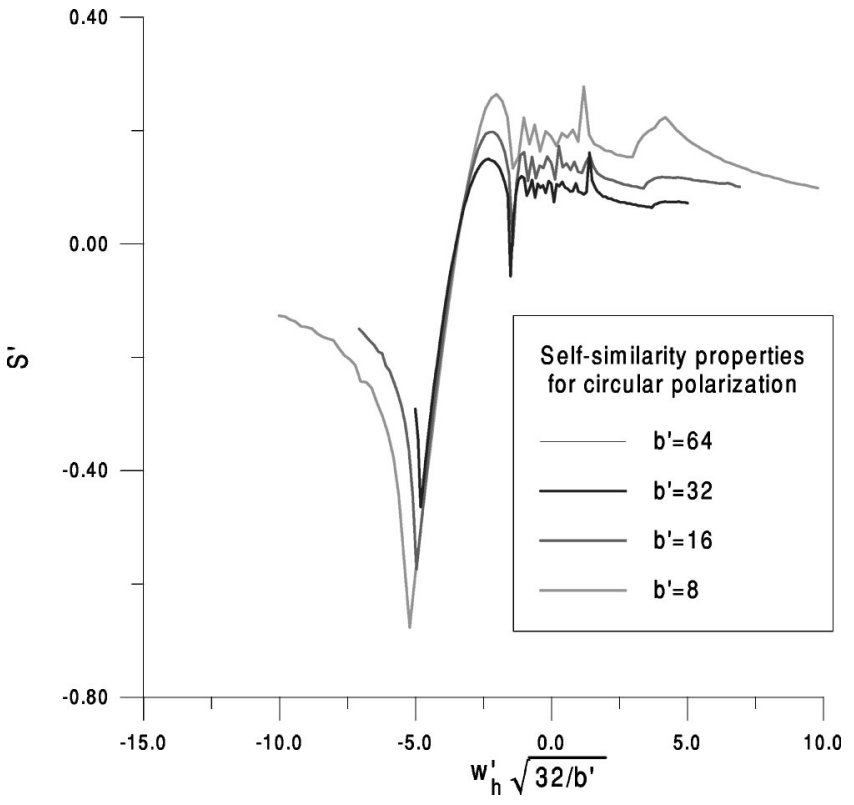

FIG. 6. Scaled stress for some values of the shear rate, as a function of the scaled frequency $w_{h}^{\prime} \sqrt{b_{0}^{\prime} / b^{\prime}}$. We have represented the case $b_{0}^{\prime}=32$, where the self-similarity of the curves becomes manifested.

For some determined values of the shear rate the induced stress has a steep increase. Consequently, the system exhibits a multiresonant response, as we can see for $w_{h}^{\prime}=0.5$ in Fig. 4. These resonances originate from the synchronization of the field with the hydrodynamic response of the system, which enhances the induction of tensions.

For a wide range of values of the frequency and a wide interval of values of the shear rate, the response of the system to the variations of the shear rate is inhibited (noresponse or "blockade" regime, corresponding to the flat curves in Fig. 4).

There also exists a regime where the transducted field energy improves the rotation of the pair in the shear, leading to a reduction of the apparent viscosity of the fluid. This phenomenon [12-15] has been referred to as the negative viscosity effect.

Finally, there appears monotonous shear thickening, shear thinning regimes or combinations of both as is manifested in Fig. 4.

It is worth pointing out that the stress curves are quite self-similar, as Fig. 6 manifests. Therefore, we can tune the regime we are interested in by properly modifying one of the parameters of the problem. Moreover, the existence of the scaling invariance ensures its accessibility in all the range of values.

\section{DISCUSSION AND CONCLUSIONS}

In this paper, we have shown the possibility of generating stresses of very different natures in assemblies of pairs of induced dipoles. The implementation of a model that mimics the dynamics of the field-responding unit leads to the appearance of a rich variety of nonlinear stress regimes involving multiresonances, shear thickening and thinning, negative viscosity or blockades. 
This multiplicity of intrinsically different behaviors together with the reversible nature of the transition mechanisms can be utilized to control the induction of stresses in the inactive phase. A broad field of applications of this phenomenon can then be open. The importance of the control of the stress lies in the fact that stress itself may induce significant modifications in soft condensed matter phases. To mention just a few examples, stresses may induce structural transitions in surfactant solutions [16] or gelation [17]; they can also modify the orientation of surfactant phases, liquid crystals [18] or polymers [19]. Moreover, alterations in the distribution of stresses may lead to important changes in the rheological properties of the system [14].

In the cases we have analyzed, possible noise sources have not been considered. Whereas absence of noise constitutes a good approximation for large particles, as magnetic holes [10], smaller particles, as in the case of a ferrofluid, are affected by Brownian torques. In the first case, the model we have proposed through Eq. (1) is enough to describe the dynamics of the suspended phase. For the ferrofluid, however, the model must and can be easily generalized to include noise sources.

Our findings may open new perspectives for research in these systems offering some insight into the mesoscopic mechanisms controlling macroscopic nonlinear behaviors.

\section{ACKNOWLEDGMENTS}

We would like to thank T. Alarcón for valuable discussions. This work has been supported by DGICYT of the Spanish Government under Grant No. PB98-1258, and by the INCO-COPERNICUS program of the European Commission under Contract No. IC15-CT96-0719. D.R. wishes to thank Generalitat de Catalunya for financial support.
[1] R.E. Rosensweig, Ferrohydrodynamics (Cambridge University Press, Cambridge, England, 1985).

[2] R.G. Larson. The Structure and Rheology of Complex Fluids (Oxford University Press, New York, 1999).

[3] J.M. Rubí and J.M.G. Vilar, J. Phys.: Condens. Matter 12, A75 (2000)

[4] P.P. Phule and J.M. Ginder, MRS Bull. 23, 19 (1998).

[5] J.E. Martin and J. Odinek, Phys. Rev. Lett. 75, 2827 (1995).

[6] H.W. Muller, Phys. Rev. Lett. 82, 3907 (1999).

[7] T.C. Halsey, J.E. Martin, and D. Adolf, Phys. Rev. Lett. 68, 1519 (1992).

[8] L. Zhou, W. Wen, and P. Sheng, Phys. Rev. Lett. 81, 1509 (1998).

[9] O. Volkova, S. Cutillas, and G. Bossis, Phys. Rev. Lett. 82, 233 (1999).
[10] G. Helgesen, P. Pieranski, and A.T. Skjeltorp, Phys. Rev. Lett. 64, 1425 (1990).

[11] A.T. Skjeltorp, in Complex Fluids, edited by L. Garrido, Lecture Notes in Physics, Vol. 415 (Springer-Verlag, Berlin, 1993), p. 243.

[12] A. Pérez-Madrid, T. Alarcón, J.M.G. Vilar, and J.M. Rubí, Physica A 270, 403 (1999).

[13] J. Happel and H. Brenner, Low Reynolds Number Hydrodynamics (Kluwer Academic, 1991), Chaps. 2 and 3.

[14] R.E. Rosensweig, Science 271, 614 (1996).

[15] J.C. Bacri, R. Perzynski, M.I. Shliomis, and G. I. Burde, Phys. Rev. Lett. 75, 2128 (1995).

[16] J. Yamamoto and H. Tanaka, Phys. Rev. Lett. 77, 4390 (1996).

[17] C. Liu and D.J. Pine, Phys. Rev. Lett. 77, 2121 (1996).

[18] C.R. Safinya et al., Science 261, 588 (1993).

[19] Z.R. Chen et al., Science 277, 1248 (1997). 